\title{
Automatic Calibration of High Density Electric Muscle Stimulation
}

\author{
JARROD KNIBBE, PAUL STROHMEIER, SEBASTIAN BORING, and KASPER HORNBAK,
}

University of Copenhagen

\begin{abstract}
Electric muscle stimulation (EMS) can enable mobile force feedback, support pedestrian navigation, or confer object affordances. To date, however, EMS is limited by two interlinked problems. (1) EMS is low resolution - achieving only coarse movements and constraining opportunities for exploration. (2) EMS requires time consuming, expert calibration confining these interaction techniques to the lab. EMS arrays have been shown to increase stimulation resolution, but as calibration complexity increases exponentially as more electrodes are used, we require heuristics or automated procedures for successful calibration. We explore the feasibility of using electromyography (EMG) to auto-calibrate high density EMS arrays. We determine regions of muscle activity during human-performed gestures, to inform stimulation patterns for EMS-performed gestures. We report on a study which shows that auto-calibration of a 60-electrode array is feasible: achieving 52\% accuracy across six gestures, with $82 \%$ accuracy across our best three gestures. By highlighting the electrode-array calibration problem, and presenting a first exploration of a potential solution, this work lays the foundations for high resolution, wearable and, perhaps one day, ubiquitous EMS beyond the lab.
\end{abstract}

CCS Concepts: • Human-centered computing $\rightarrow$ Ubiquitous and mobile computing systems and tools;

Additional Key Words and Phrases: ACM proceedings; Electric Muscle Stimulation; Calibration

ACM Reference format:

Jarrod Knibbe, Paul Strohmeier, Sebastian Boring, and Kasper Hornbæk. 2017. Automatic Calibration of High Density Electric Muscle Stimulation. Proc. ACM Interact. Mob. Wearable Ubiquitous Technol. 1, 3, Article 68 (September 2017 ), 17 pages.

DOI: http://doi.org/10.1145/3130933

\section{INTRODUCTION}

Electric Muscle Stimulation (EMS) has emerged as an output paradigm in HCI, whereby electrical current is applied to a muscle, causing muscle actuation. EMS has been used to provide force feedback for mobile games [14], confer object affordances [18], teach musical instruments [33], support sketching [19], and assist in pointing [10]. If we wish to move beyond these special purpose interactions, towards general purpose computer-human control, we need to address two outstanding challenges in using EMS in HCI: (1) resolution and (2) calibration. Outside HCI, EMS arrays have been shown to increase resolution (e.g., [27,28]). However, increased resolution requires exponentially more expert and time-consuming calibration procedures, which has constrained the wider exploration and deployment of EMS. In turn, these challenges have prevented the adoption of EMS as an interaction technique for ubiquitous computing, with a dearth of papers at Ubicomp exploring deployed EMS systems. We explore an auto-calibration technique for high density EMS arrays. We seek to reduce the time requirements and remove any need for expertise in calibration, making available electrode arrays and higher resolution stimulation for $\mathrm{HCI}$ in the future.

\footnotetext{
Permission to make digital or hard copies of all or part of this work for personal or classroom use is granted without fee provided that copies are not made or distributed for profit or commercial advantage and that copies bear this notice and the full citation on the first page. Copyrights for components of this work owned by others than the author(s) must be honored. Abstracting with credit is permitted. To copy otherwise, or republish, to post on servers or to redistribute to lists, requires prior specific permission and/or a fee. Request permissions from Permissions@acm.org. 2474-9567/2017/9-ART68 \$15.00 Copyright is held by the owner/author(s). Publication rights licensed to ACM. DOI: http://doi.org/10.1145/3130933
} 
68:2 J. Knibbe et al.

\subsection{EMS is Low Resolution}

Existing work using EMS for HCI has relied on low resolution stimulation; supporting both a low number of gestures and providing a coarse granularity of induced motion. The presented systems are typically special purpose, requiring the repositioning and recalibration of electrodes if the user wishes to transition from inferring object affordances [18] to receiving assistance in drawing [19], for example. Additionally, existing systems typically use only coarse motion such as wrist flexion, extension and pronation [18].

The challenge surrounding resolution stems from two factors that make targeting desired muscles difficult. Firstly, the anatomy of the forearm is complex, with muscles both tightly packed and layered. Secondly, transcutaneous stimulation disperses unpredictably into the underlying tissue and muscle. Literature in the medical sciences has demonstrated that high density EMS can go some way to addressing these challenges, without the need for invasive needle-based stimulation. Using multiple, uniquely shaped electrodes increases resolution, improves comfort and decreases fatigue [27,28]. This enables stimulation of more precise movements, while simultaneously increasing the range of possible motion. While high-density electrode arrays have been used for surface electromyography (HD-sEMG) research in HCI [1], this approach is yet to be explored for EMS. We draw inspiration from the literature demonstrating the potential of EMS arrays, and attempt to facilitate their use in HCI through auto-calibration.

\subsection{EMS Requires time-consuming, Per-User Calibration}

While the interaction techniques presented in HCI are many, varied and novel, their exploration is currently limited to a laboratory setting due to time-consuming and expert calibration requirements. To use EMS in interaction design, EMS-designers must engage in both spatial calibration, correctly positioning the electrodes, and signal calibration, tuning stimulation parameters, to target correct muscle depths and minimize discomfort. A typical calibration process may proceed as follows: the EMS-designer first determines their target gesture and from there identifies the required muscles. They may choose to do this anatomically, such as by using muscle diagrams, or through visual or tactile inspection of arm-surface deformations during muscle contractions (e.g., palpation). As not all muscles are targetable individually, not all gestures are available to the designer. Thus, the EMS designer engages in a back and forth, trial-and-error led procedure, where electrodes are placed, tested, repositioned, tested, and so on. This is spatial calibration. Simultaneously to this process, the designer will be testing a range of signal parameters, varying the pulse-width, frequency and amplitude at each new electrode position, as these parameters can change the resultant movement. This is signal calibration.

Once approximate electrode positions and signal parameters are determined, these can then be used as a guide with which to calibrate each individual user. Due to individual differences, including depth of fat, muscle size, skin resistance, etc., the exact electrode locations and signal parameters will vary per user. This process is time-consuming, taking up to five minutes per participant per desired pose or gesture when only positioning a small number of electrodes [18]. If a complex set of gestures is required for the interaction technique, such as the six poses used in Affordance++ [18], then the required time for calibration can quickly become impractical - requiring up to 30 minutes of setup prior to every use.

As we move towards array-based EMS, the challenge changes from where to place individual electrodes, to which electrodes in the array to employ. To make full use of EMS arrays' potential, multiple electrodes need to be selected and coordinated to provide stimulation. This exponentially increases the available permutations of electrode combinations and thus also the complexity of spatial-calibration.

As a result of the complexity of calibration, the opportunities for widespread, longitudinal 'out-of-the-lab' exploration are limited. As a community, we have seen that EMS-interactions are compelling in the lab, but deploying these systems in the wild remains challenging.

Proceedings of the ACM on Interactive, Mobile, Wearable and Ubiquitous Technologies, Vol. 1, No. 3, Article 68. Publication date: September 2017. 


\subsection{Contribution: Towards Auto-Calibration of High-Density EMS}

Our paper is the first attempt at automatic spatial calibration for high density EMS for HCI. We explore the use of EMG to auto-calibrate a 60-electrode array for EMS. Our goal is to enable a user to simply apply the electrode array and, while EMG data is collected, perform a gesture that they wish to use for their interaction technique. The system then identifies the electrode configuration required to perform this gesture with EMS (Fig. 1). To achieve this, the EMG data is read across all electrodes and processed to provide a mapping for EMS stimulation of the same gesture. Through auto-calibration, we seek to make high density EMS practical and facilitate higher resolution stimulation for HCI.

We report on a study of the mapping between EMG and EMS. Although EMG and EMS have been previously combined for single location, temporal synchronization in HCI [25], we present the first implementation of their use for spatial auto-calibration. This has been approached in the rehabilitation literature [5], but here we explore calibration of higher complexity, multi-electrode patterns.

In our study, we achieve an average of $52 \%$ replay accuracy over six gestures, with $82 \%$ calibration accuracy on average across our best three gestures. Our most accurate gestures include our most complex gesture; the accurate control of the index finger separately from the other fingers. Overall, we achieve $67 \%$ correct identification of target muscle compartments.

To enable our study, we developed a prototype array and multiplexer circuitry. The array is designed as a sleeve, to support easy application and removal across a wide range of arm sizes. The array consists of 60 electrodes. Where typically in HCI electrodes are used 1:1 (anode:cathode) for EMS, our sleeve can write any combination of $\mathrm{p}: \mathrm{q}$ electrodes (where $\mathrm{p}, \mathrm{q} \geq 1$ ). This explodes the possible electrode mappings to $3^{n}-2^{n+1}+2$ configurations (where $n$ is the number of electrodes). This facilitates higher resolution stimulation, but makes proven calibration methods, such as the one introduced in PossessedHand [33], infeasible.

Schematics and source code for our prototype are provided ${ }^{1}$. We discuss the current limitations of our approach, including the non-interactive rate of calibration and, from this, the calibration of poses rather than dynamic gestures. We detail future refinements for further improving calibration accuracy. The prototype sleeve is designed as a motivating vision of a future of wearable, ubiquitous EMS, made possible through autocalibration.
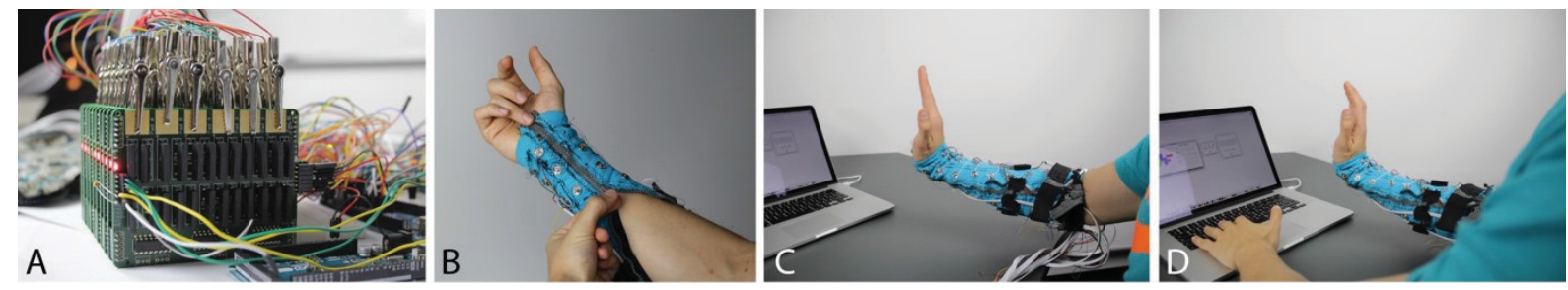

Fig. 1. A) Custom signal switching boards to multiplex EMG and EMS signals between 60 electrodes. B-D) The process of EMG-calibration for EMS. B) The user puts on the sleeve. C) The user performs a desired pose whilst EMG data is gathered. D) An EMS stimulation pattern is calculated from the EMG data. The user can then explore the signal parameters across that stimulation pattern.

\section{RELATED WORK}

\subsection{EMS in $\mathrm{HCl}$ : Time-Intensive, Expert Calibration}

EMS provides rich opportunities for computer-human control, such as providing muscle propelled force feedback for mobile and VR gaming [14-16], providing guidance in everyday activities such as walking or

\footnotetext{
${ }^{1}$ https://github.com/jarrodknibbe/EMS_AutoCalibration
} 
drawing [19,26], and exploring proprioceptive interaction and the interplay between muscle sensing and stimulation [17]. Across this literature, spatial calibration involves manually placing the electrodes and observing the resulting actuation, while signal calibration is done based on self-reports by the study participant. In Affordance++ [18], for example, Lopes discusses pre-study, per-participant calibration of placement, pulse-width, pulse frequency, and amplitude. They report a calibration time of two to five minutes per participant per pose. Therefore, the use of six poses in their work quickly sums to a lengthy calibration procedure. In Cruise Control for Pedestrians [26], Pfeiffer reports on a calibration study, highlighting the differences in stimulation parameters (current and voltage) both between participants and between individual participants' legs. Furthermore, Pfeiffer suggests the need for spatial-calibration accuracy of $+/-5 \mathrm{~mm}$ to achieve the desired effect.

When using multi-electrode setups, the spatial calibration process changes. Instead of manually determining the correct location for stimulation by placing, removing and reapplying the electrodes, the electrode positions are fixed. Thus, the spatial calibration process selects which of the multiple electrodes will be used. This approach was demonstrated by Tamaki et al. with PossessedHand [33]. Tamaki et al. applied stimulations across 12 different voltage levels through a band consisting of 14 electrode pairs. In perparticipant calibration, the system runs through all pairs of electrodes for all voltage levels, resulting in 168 stimulations. During this process, the operator clicks the effected joint locations on a GUI in order to train the system. This achieves both spatial and signal calibration, and remains the state-of-the-art in HCI.

More recently, Tamaki et al. explored using physiological data to improve this calibration process in UnlimitedHand [32]. They presented a system that uses photo-reflectors to observe muscle displacements under contraction. This data is processed to drive stimulation across eight unique electrodes, pre-designed for their assumed position on the arm. This allows for fast calibration - four gestures were calibrated in ten seconds. The requirement for additional on-skin sensors, however, reduces the maximum skin coverage available for electrodes, in turn reducing the overall resolution. Furthermore, by designing the electrodes based on the assumed position of the muscles and requiring a fixed position for the device, the technique is less adaptable to per-user variations in muscle size and location, and more susceptible to problems due to incorrect positioning. For example, Popovic-Bijelic et al. highlight the differences in electrode configurations required to achieve the same movement in three participants [28].

Physiological sensors do not necessarily require concessions in spatial resolution, however. Electromyography (EMG), for example, can employ the same electrodes that are used for EMS. Building on the work of Shalaby et al. [29], Nishida demonstrated a technique for reading EMG between EMS stimulation pulses [25]. They demonstrated using voluntary muscle contractions to trigger stimulation, for example.

Our work explores EMG for spatially-calibrating an EMS array. By doing so we avoid the necessity for manual positioning and repositioning of electrodes as required by the systems of Lopes et al. and Pfeiffer et al. We overcome the limitations of UnlimitedHand [32] by using a design with increased spatial coverage, and thus resolution. While the calibration method presented by Tamaki et al. in PossessedHand works well for a limited number of electrodes [33], it does not generalize to a larger electrode array. PossessedHand requires testing of 14 electrode pairs, while the lower bound of electrode combinations that need to be tested in our system is $\sim 10^{18}\left(2^{n}-2\right.$, where $\mathrm{n}$ is the number of electrodes). While UnlimitedHand provides a possible direction for calibrating a larger array [32], the spatial separation of sensing and stimulation methods limits the available stimulation resolution. Instead, we maintain high spatial resolution by using the same electrodes for both sensing and stimulation, as suggested by Shalaby et al. [29] and Nishida et al. [25]. As our research focuses on the feasibility of using EMG for spatial-calibration of EMS, we chose not to implement the concurrent EMG-EMS that Shalaby et al. and Nishida et al. detail. When applied to an array such as ours, Shalaby et al.'s and Nishida et al.'s techniques quickly become very technically complex, representing a different problem that is not the focus of our work. We acknowledge that, in future work, these techniques could be used to speed up the calibration process.

We present an auto-calibration technique for a dense array of multi-electrode, multi-signal stimulation. Our approach is both rotation and position invariant, making it easy to apply in lab settings as well as in the 
wild'. Duente et al. provide a motivating vision for the future of combined muscle sensing and actuation technologies in their workshop paper [6]. We provide the first realization of this vision.

\subsection{EMS Beyond HCl: High resolution, Complex Calibration}

Beyond HCI, bio-medical research on rehabilitation has explored electrode arrays and wearable electrode solutions, including dense electrode designs (e.g., [11]) and personalized layouts [20]. These arrays are capable of providing targeted stimulation that reduces movement artifacts, fatigue and discomfort (e.g., [20,27]). Although these works present calibration techniques, they still focus on (a) walk-throughs of electrode combinations (e.g., [9,21]), that can quickly scale to impractical iterations, (b) calibration of arrays consisting of only few or single-purpose electrodes (separating sensing and stimulation electrodes) [7], or (c) complex training procedures for neural-activity control [3], that are infeasible for more generalized HCI domains. More recently, De Marchis et al. explored a combination of EMG and EMS across a 30-electrode array [5]. They focused their calibration on pair-wise stimulation of three different muscles, using precisely placed, small surface area electrode arrays. Their results demonstrate the promise of the technique, with $66 \%$ motion reproduction accuracy.

In this paper, we build upon the work of De Marchis et al. by expanding it beyond pair-wise calibration, allowing for complex, multi-electrode stimulation configurations. However, the HCI context also presents additional design considerations, such as the ease of use and accessibility to interaction-designers. We address these by calibrating per-pose rather than per-muscle. This means that users can apply the electrode array without the requirement for precise alignment and EMS-designers can design interaction techniques without the need for in-depth physiological knowledge. Finally, our system spans the entirety of the forearm, covering many more muscle groups and opening the potential for many more gestures to be calibrated than in existing systems.

\subsection{Arrays For EMG}

We explore the use of EMG to calibrate for EMS. Note that we claim no novelty or contribution in EMG. Here we present the work that helped inspire our prototype implementation and could drive future refinements.

EMG research has recently been exploring wearable form-factors (e.g., [2,23]) and dense arrangements (e.g., [1,8]). Amma et al. explored an EMG sensor array for finger gesture recognition [1]. Their prototype consisted of 192 electrodes and achieved high classification accuracy ( $90 \%$ across 27 gestures) - demonstrating the benefits of a dense electrode arrangement. Amma et al. used an array with $10 \mathrm{~mm}$ inter-electrode spacing. Electrodes of this size can easily result in a 'burning' sensation when used for EMS, as a result of currentdensity, so stimulation would need to be spread across multiple electrodes. Amma et al. suggest that although higher electrode numbers are beneficial, between 20 and 80 represent a good trade-off between accuracy and technical complexity. From their recommendation, we use $60 \sim 20 \mathrm{x} 15 \mathrm{~mm}$ electrodes, while maintaining minimal inter-electrode spacing (as also suggested in [13]).

\begin{tabular}{|c|c|c|}
\hline Research & $\begin{array}{l}\text { No. of } \\
\text { Electrodes } \\
\text { (total) }\end{array}$ & Calibration Details Provided \\
\hline PossessedHand [34] & 28 & $\begin{array}{l}\text { Automatically run all signal configurations across all electrode pairs (168 } \\
\text { combinations). The user selects joints under movement on an interface. We } \\
\text { estimate this takes } \sim 14 \text { minutes ( } 5 \text { seconds for stimulation and GUI selection, per } \\
\text { combination). }\end{array}$ \\
\hline
\end{tabular}

Feedback [12]

$4 \quad$ Participants were calibrated to produce visible, yet pain free movement.

Proceedings of the ACM on Interactive, Mobile, Wearable and Ubiquitous Technologies, Vol. 1, No. 3, Article 68. Publication date: September 2017. 
68:6 J. Knibbe et al.

\begin{tabular}{|c|c|c|}
\hline Research & $\begin{array}{l}\text { No. of } \\
\text { Electrodes } \\
\text { (total) }\end{array}$ & Calibration Details Provided \\
\hline Affordance $++[16]$ & 6 & $\begin{array}{l}\text { A total of } 6 \text { different poses/motions were used. Calibration of each motion took 2- } \\
5 \text { minutes. (Potentially } 30 \text { minutes for all motions, in the worst case). Calibration } \\
\text { was performed once per user and ensured no pain was felt. }\end{array}$ \\
\hline $\begin{array}{l}\text { Proprioceptive } \\
\text { Interaction [15] }\end{array}$ & 4 & $\begin{array}{l}\text { Participants self-calibrated, reporting the minimum motion-causing stimulation } \\
\text { and the maximum comfortable stimulation. }\end{array}$ \\
\hline Muscle Plotter [17] & 8 & $\begin{array}{l}\text { The paper reports on electrode placement, signal calibration and re-calibration } \\
\text { (due to fatigue). Incorporates 3-step calibration process into study: } \\
\text { 1: Determine Comfortable use - finding minimum stimulation for movement and } \\
\text { maximum comfortable. } \\
2 \text { \& 3: Calibrate the signal for achieving certain movements whilst drawing. }\end{array}$ \\
\hline $\begin{array}{l}\text { Cruise Control for } \\
\text { Pedestrians [25] }\end{array}$ & 4 & $\begin{array}{l}\text { Reports the need for accurate calibration, suggesting }+/-5 \mathrm{~mm} \text { from muscle body, } \\
\text { to prevent co-activation of additional muscles or no activation. Muscles selected } \\
\text { that prevented 'awkward' electrode placement. }\end{array}$ \\
\hline Let Me Grab This [26] & 4 & $\begin{array}{l}\text { Authors initially pretested device on themselves to remove any 'pain-causing' } \\
\text { signal combinations. These were applied to the participants. (No per-participant } \\
\text { signal calibration conducted.) }\end{array}$ \\
\hline Follow the Force [9] & 8 & $\begin{array}{l}\text { Fixed signal parameters were determined in a pre-study test. This was conducted } \\
\text { to reduce complexity and 'keep the calibration time at an acceptable level.' The } \\
\text { participants slowly increased the EMS intensity themselves, until reaching the } \\
\text { limits of comfortable signal strengths. }\end{array}$ \\
\hline $\begin{array}{l}\text { 3D Virtual Hand } \\
\text { Selection with EMS } \\
\text { and Vibration } \\
\text { Feedback [27] }\end{array}$ & 2 & $\begin{array}{l}\text { Pulse width and frequency were selected from a previous study, where they were } \\
\text { found 'suitable' for a large range of users. The intensity of the EMS was calibrated } \\
\text { for each individual user, to account for skin resistance and variations in } \\
\text { contraction effect. }\end{array}$ \\
\hline UnlimitedHand [33] & 8 & $\begin{array}{l}\text { Photo-reflectors sense muscle displacement under contraction. This data then } \\
\text { drives electrode selection for gesture replication. Calibration of four gestures } \\
\text { takes ten seconds, including stimulation across } 6 \text { different stimulation parameters. }\end{array}$ \\
\hline $\begin{array}{l}\text { Let Your Body Move } \\
\qquad[24]\end{array}$ & $\mathrm{N} / \mathrm{A}$ & $\begin{array}{l}\text { Provides a detailed guide for calibrating participants. Participants increase } \\
\text { stimulation until the maximum comfortable. After this, further muscles should be } \\
\text { located. Details participant differences based on tissue thickness, body fat } \\
\text { percentage, skin moisture, and hair. }\end{array}$ \\
\hline $\begin{array}{l}\text { New Multi-Channel } \\
\text { Transcutaneous } \\
\text { Electrical Stimulation } \\
\text { Technology for } \\
\text { Rehabilitation [10] } \\
\end{array}$ & $\begin{array}{l}60 \text { (array } \\
\text { style) }\end{array}$ & $\begin{array}{l}\text { An additional larger cathode electrode was placed below the array (closer to the } \\
\text { hand). The electrodes of the array were stimulated in a random order and the } \\
\text { resultant grip force for each finger was measured using strain gauges. A rest } \\
\text { period was maintained between stimulations. The process took, in total, } \\
\text { approximately six minutes. }\end{array}$ \\
\hline $\begin{array}{l}\text { Optimal Selection of } \\
\text { Electrodes for Muscle } \\
\text { Electrical Stimulation } \\
\quad \text { Using Twitching } \\
\text { Motion Measurement }\end{array}$ & 4 & $\begin{array}{l}\text { Four unique combinations of the electrodes were stimulated in turn. A motion } \\
\text { capture device measures the resultant movement from each electrode } \\
\text { configuration. The electrodes providing the maximum movement in a given } \\
\text { direction is determined. }\end{array}$ \\
\hline
\end{tabular}

[8]

Proceedings of the ACM on Interactive, Mobile, Wearable and Ubiquitous Technologies, Vol. 1, No. 3, Article 68. Publication date: September 2017. 


\begin{tabular}{|c|c|c|}
\hline Research & $\begin{array}{l}\text { No. of } \\
\text { Electrodes } \\
\text { (total) }\end{array}$ & Calibration Details Provided \\
\hline $\begin{array}{l}\text { Muscle twitch } \\
\text { responses for shaping } \\
\text { the multi-pad electrode } \\
\text { for function electrical } \\
\text { stimulation [19] }\end{array}$ & $\begin{array}{l}16(+1 \\
\text { large } \\
\text { cathode at } \\
\text { wrist })\end{array}$ & $\begin{array}{l}\text { Two different protocols: } \\
\text { Twitch protocol: single pulse was delivered nine times (at } 2 \mathrm{~Hz}) \text { to each pad. An } \\
\text { accelerometer measured movement at the back of the hand and at the finger. } \\
\text { Continuous: } 30 \mathrm{~Hz} \text { pulse trains were delivered for two seconds. Joint angles were } \\
\text { measured with goniometers at the back of hand and fingers. }\end{array}$ \\
\hline $\begin{array}{l}\text { A multi-pad electrode } \\
\text { based functional } \\
\text { electrical stimulation } \\
\text { system for restoration } \\
\quad \text { of grasp [18] }\end{array}$ & $\begin{array}{l}16 \text { (in } \\
\text { study) }\end{array}$ & $\begin{array}{l}\text { Uses the twitch protocol, as above, to build a lookup table of electrode selection } \\
\text { and effect. Infers combinations of electrodes for targeting specific muscles and } \\
\text { end-effectors. }\end{array}$ \\
\hline $\begin{array}{l}\text { Restoring cortical } \\
\text { control of function } \\
\text { movement in a human } \\
\text { with quadriplegia [3] }\end{array}$ & 130 & $\begin{array}{l}\text { The user controlled the electrodes through a neural bypass system (NBS), } \\
\text { implanted in his motor cortex. The user attended } \leq 3 \text { sessions per week }(\sim 3.5 \mathrm{hrs} \\
\text { per session) for } 15 \text { months to calibrate the implant/electrode sleeve system. }\end{array}$ \\
\hline $\begin{array}{l}\text { Multi-Contact } \\
\text { functional electrical } \\
\text { stimulation for hand } \\
\text { opening [5] }\end{array}$ & 30 & $\begin{array}{l}\text { Uses EMG to calibrate an EMS array. Only interested in pairwise stimulation of } \\
\text { three different muscles. Provides stimulation to only one maximal pair. }\end{array}$ \\
\hline Our Work & 60 & $\begin{array}{l}\text { Users perform a desired gesture. During this time, EMG data is collected } \\
\text { between } 306 \text { unique electrode combinations. This EMG data is used to } \\
\text { derive complex, multi-electrode patterns for EMS. Multi-channel } \\
\text { stimulation is calibrated. Calibration takes } \sim 1 \text { minute per pose. }\end{array}$ \\
\hline
\end{tabular}

\section{HIGH-DENSITY EMS: PROTOTYPE AND CALIBRATION PROCESS}

In order to explore the use of EMG to spatially calibrate an electrode array for EMS, we designed and built a prototype electrode array sleeve and custom signal switching circuitry.

\subsection{A High-Density Electrode Sleeve}

The high-density electrode sleeve is constructed on a cotton sports compression sleeve. Sixty $\sim 2 \times 1.5 \mathrm{~cm}$ electrodes were sewn into the sleeve in a $6 \times 10$ grid. The electrodes were cut from conductive fabric and interface to the outside of the sleeve with metal poppers. A layer of electrode hydrogel (AxelGaard's AG635 'Sensing' Hydrogel) ensures a good connection between the conductive fabric and the skin. Currently, the gel is cut into per-electrode pieces. While time-consuming, this improves the muscle 'selectivity' of our electrodes. One large sheet of electrode gel could also be used here, however, this would serve to distribute the stimulation across a wider area, decreasing between-electrode effects, but reducing the targeting resolution [12]. For ease of application a zip was added to the side of the sleeve. The sleeve can be put on and removed by the wearer. As a result of the design of the sleeve, the zip is typically worn down the center of the inner forearm (as the wearer can hold the top of the zip with their wearing-hand, while pulling the zipper with their other hand). Given the size of the electrodes and the use of multiple-electrode patterns, the sleeve goes some way towards supporting a one-time calibration process (as the re-applied electrodes typically closely align with their initial position). With a more tailored design, such as the use of a 'thumb loop' or clear 'elbow patch', this could be further ensured.

The initial sports compression sleeve spanned the wrist to the mid-bicep. As a result of the additional materials we added (especially the zip), our electrode sleeve covers the wrist to the elbow. 
The sleeve is connected to the switching circuitry by $1.5 \mathrm{~m}$ of cable. This supports a wide range of motion when wearing the sleeve. Future iterations can be made fully wearable (achieving miniaturization through surface mount components, for example), supporting exploration on the tennis court, when moving around the house, whilst cooking, etc.
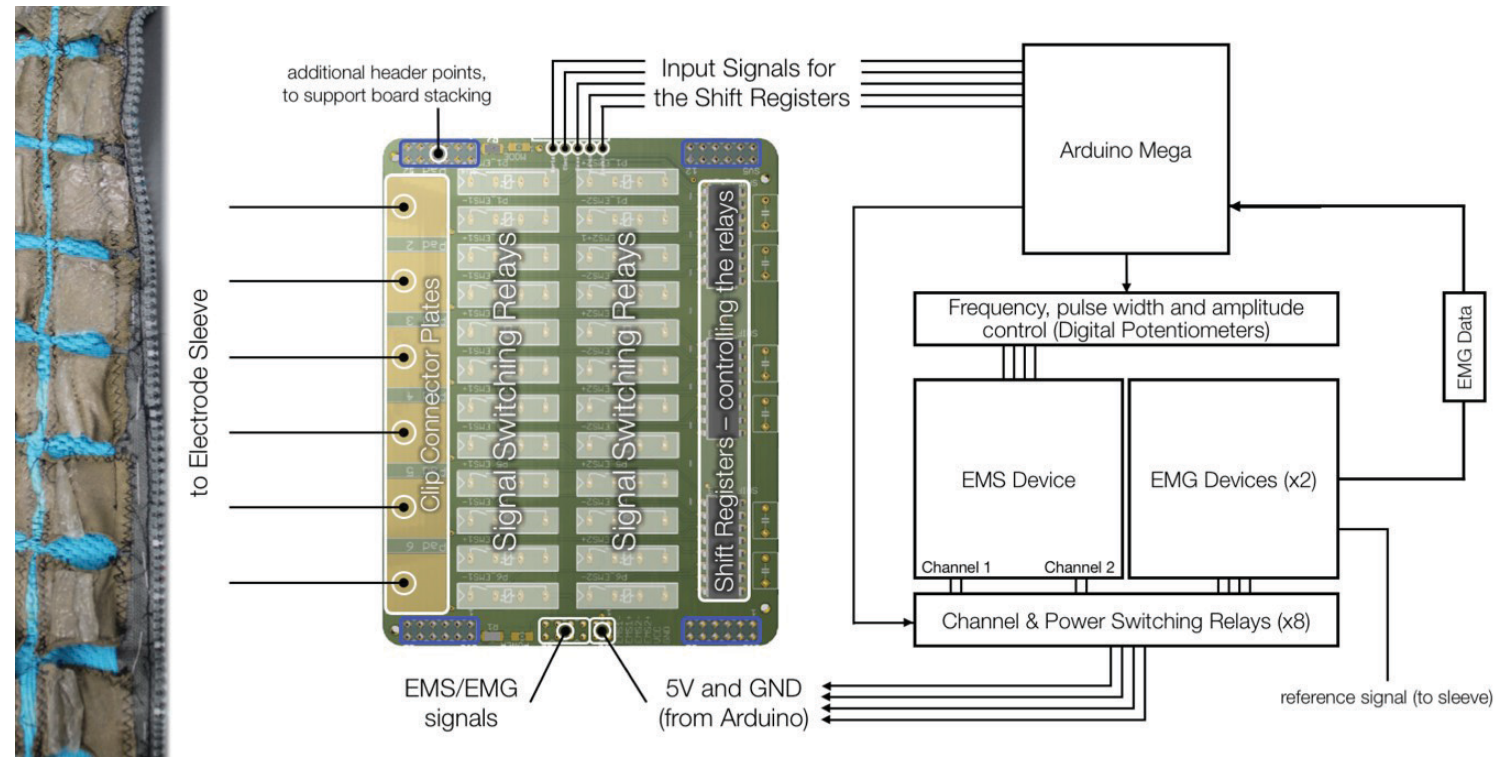

Fig. 2. Illustration of the prototype circuitry design. An Arduino routes the signal from an EMS device and two EMG devices onto custom signal switching boards. The switching boards route the signals onto any combination of 60 electrodes sewn into a sleeve.

\subsection{Custom Electronics}

We designed and built custom circuitry to multiplex both EMG and EMS signals across our sleeve (see Figures 1 and 2). Our design is based around two off-the-shelf EMG devices (Backyard Brains, Muscle Spiker shields) and an EMS device (Med-Fit 1, dual channel Tens machine ${ }^{2}$ ). This EMS device provides control of signal amplitude, pulse width and frequency. We control these parameters through 2 digital potentiometers (DS1803050, 2 channel, 256-step, $50 \mathrm{k} \Omega$ ). The amplitude was originally manually controlled through two $20 \mathrm{k} \Omega$ potentiometers, thus we have 102-steps of amplitude resolution control.

We designed custom PCBs to control the signal-electrode switching. Each signal switching board routes the signals, in any combination, to 6 electrode connectors (for one row of our sleeve). The signals are routed

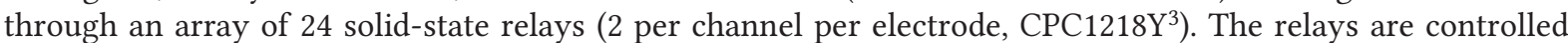
through 3 chained 8-bit shift registers (SN74HC595N). We use 10 signal switching boards in total (for our 10x6 electrode sleeve), stacked through connecting headers into a tower (240 relays in total). Additional relays switch the inputs to the signal switching boards between EMG and EMS, and power on/off the EMG and EMS devices as needed, to prevent any potential electrical damage. NO relays (normally open) ensure no stimulation signal connection to the wearer, should an error occur or the switching board power fail.

\footnotetext{
${ }^{2}$ http://www.med-fit.co.uk/tens-and-stimulation/med-fit-1.html

${ }^{3} \mathrm{We}$ chose solid state relays rated to $60 \mathrm{~V}$. While our EMS device provides $40 \mathrm{~V}$ maximum at $500 \mathrm{Ohm}$, the current driven signal can exceed $60 \mathrm{~V}$ at skin-level resistance. This high-level voltage only occurs in brief voltage spikes. We tested our relays extensively and found no failure behavior. However, we would suggest a relay with a higher maximum voltage in the future (such as the CPC1215).
}

Proceedings of the ACM on Interactive, Mobile, Wearable and Ubiquitous Technologies, Vol. 1, No. 3, Article 68. Publication date: September 2017. 
The custom signal switching boards and circuitry are driven through an Arduino Mega (requiring 56 digital pins and 2 analog pins). An additional $5 \mathrm{~V}$ power supply is used to support the current requirements of the switching boards. The Arduino is interfaced with Python, to provide a GUI for control. Full schematics and control source code are available online ${ }^{4}$.

\subsection{Muscle Reading}

We use bipolar EMG to read muscle action potentials during muscle actuation. Where our prototype implementation supports multi-electrode selection for complex stimulation patterns, adding additional electrodes to the EMG reading adds noise to the signal. Previous work from both physiology and HCI demonstrates that the optimal configuration for EMG electrodes is above and below the center of the muscle, placed 10-30mm apart [1,8,22]. Additionally, not all muscle fibers tend downwards (for example pennate muscles, such as the flexor carpi ulnaris in the forearm), so we do not only read directly down the sleeve. Readings are taken between each electrode and the 3 closest electrodes in the next two rows for a total of 306 unique pairings (see Fig. 3). Reading starts from the top of the sleeve, nearest the shoulder, and continues downwards towards the wrist, following the muscular anatomy of the forearm. The reference electrodes are placed on the biceps brachii.

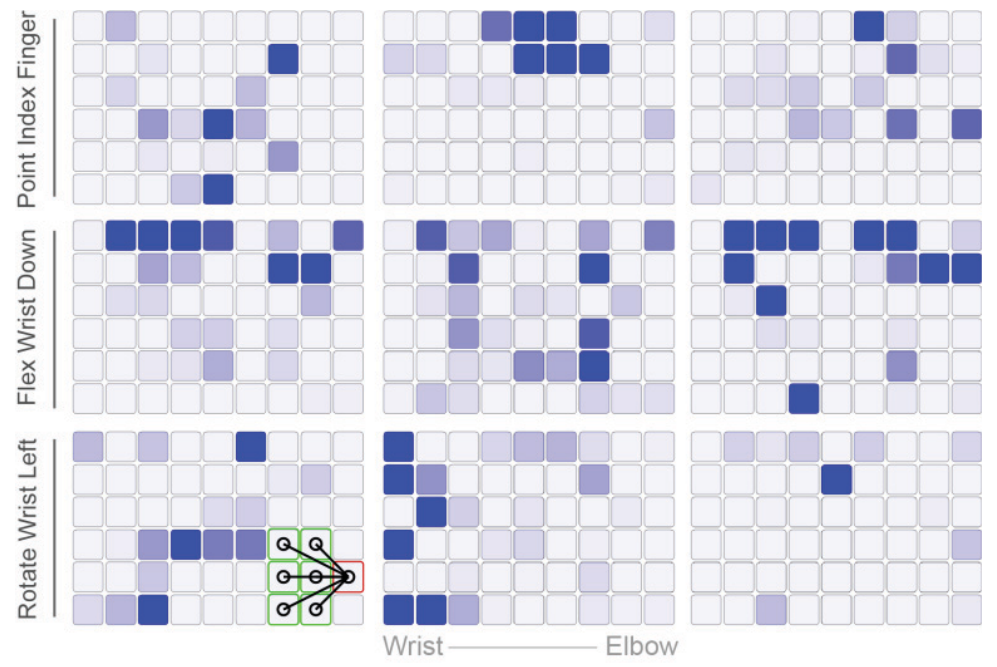

Fig. 3. Individual differences in EMG patterns across 3 users and 3 example gestures. Bottom Left: illustrates the EMG electrode reading patterns, between red (anode) and green (cathode) electrodes.

Our circuitry uses relays to select between different electrode pairs. The process of switching EMG signals between electrodes adds a spike to the EMG signal. As a result, each EMG pair is read for 220ms and only the data from the last $20 \mathrm{~ms}$ is used. Currently, based on using two EMG devices concurrently, a full reading cycle takes $\sim 45$ seconds (for 306 unique electrode pairs). During this cycle, the participant must actively hold the desired pose (i.e., maintaining muscle tension). Currently, this results in the classification of poses, not dynamic gestures. This reading time can be shortened by combinations of (a) customized EMG hardware (allowing signal rectification in code not hardware), (b) using more EMG channels simultaneously (however, this introduces additional hardware complexity), or (c) exploring feature vector patterns from dynamic gesture data, as in [34].

\footnotetext{
${ }^{4}$ https://github.com/jarrodknibbe/EMS_AutoCalibration.
} 


\subsection{Muscle Writing}

Previous research on EMS interaction techniques has focused on the use of pairwise electrodes for stimulation, writing 1:1 from an anode to a cathode (e.g. [14,18]). EMS arrays, however, can support complex patterns of electrodes to be used for EMS, resulting in $\mathrm{p}$ : q electrode configurations, where $\mathrm{p}, \mathrm{q} \geq 1$, forming electrodes of arbitrary shape and size. Our hardware setup supports multi-channel, and thus multi-amplitude EMS. This enables primary and secondary stimulation patterns, as in Muscle Plotter [19], where the primary actuation is controlled by one channel and causes the principle motion, with a second channel building upon or subtracting from this motion. For example, the primary electrodes may pull the hand up at the wrist, whilst the secondary electrodes provide small alterations to finger positions. Alternatively, agonist (primary) and antagonist (counter) muscles can be targeted separately. Combining electrodes also serves to distribute the stimulation signal across a larger surface area. As a result of this, the user may choose to increase the EMS amplitude beyond that typical if using a pairwise configuration.

\subsection{Walkthrough: the EMG - EMS Mapping Process}

The user puts on the sleeve and EMG data for an initial 'rest' pose is read as a baseline from which to normalize future poses. The normalization helps mitigate the effect of muscle activity involved in the rest pose. The user then performs and holds a desired pose whilst EMG data is collected. The root mean square (RMSQ), standard deviation and signal peak maximum are calculated for each electrode pairing. These values are standardized against the corresponding values from the rest pose. The electrodes in each pair are labelled - anode and cathode - and then sorted into 3 groups using k-means clustering. The three clusters are 1) inactive, 2) low potential, and 3) high potential. The clustering is based on our use of 2 stimulation channels. Both the anode and cathode electrodes are added to the calculated cluster. As individual electrodes occur in multiple pairings, for example each anode is paired with six cathodes (see Fig. 3), it is possible that any one electrode can be placed in multiple clusters. In this case, the electrode in the highest cluster is prioritized. Within each cluster, the electrode pairs are sorted by RMSQ magnitude. Electrodes are finally selected as anode, cathode, or 'off' based on their highest cluster and highest magnitude pairing. This electrode assignment is subsequently used for EMS.

An amplitude ratio between the stimulation channels is automatically calculated from the average RMSQ of each cluster. This ratio is balanced with the number of electrodes in each cluster as the current per electrode decreases with increasing electrode count. Once calculated, the stimulation pattern is presented to the user through a GUI, and the system automatically maintains the amplitude ratio between the EMS channels. The user can then explore the amplitude, pulse width and frequency of the pattern either through keyboard shortcuts or with the mouse.

\section{STUDY: SPATIAL-CALIBRATION OF EMS ARRAYS WITH EMG}

We ran a study to explore the use of EMG to automatically spatially-calibrate EMS arrays. Our study focuses on the calibration of multi-electrode patterns for EMS, stimulating between any number of anodes and cathodes with two signal sources. As no comparable techniques exist for multi-electrode EMS array calibration, we ran an exploratory study. As a proof of our calibration concept, we study auto-calibration of an electrode array for gestures typically used in the HCI EMS literature.

\subsection{Procedure}

Nine participants put on the electrode array and performed six gestures previously used in the EMS literature (plus an additional rest gesture for EMG signal base-lining). These gestures include: wrist flexion (wrist-down) [17], wrist extension (wrist-up) [18], radial deviation (hand rotation towards the thumb, hand-left) [10], ulnar deviation (hand rotation towards the little finger, hand-right) [10], squeeze-fingers [18], and lift index finger [10] (Fig. 4). Although electrode arrays may be used to facilitate a higher resolution of control through

Proceedings of the ACM on Interactive, Mobile, Wearable and Ubiquitous Technologies, Vol. 1, No. 3, Article 68. Publication date: September 2017. 
stimulation [20,27], our intention here is to validate the use of EMG for auto-calibration. For this reason, and to support clearer reflection on the calibration processes previously discussed, we chose to use existing gestures from the literature. The order of the gestures was counterbalanced across participants.
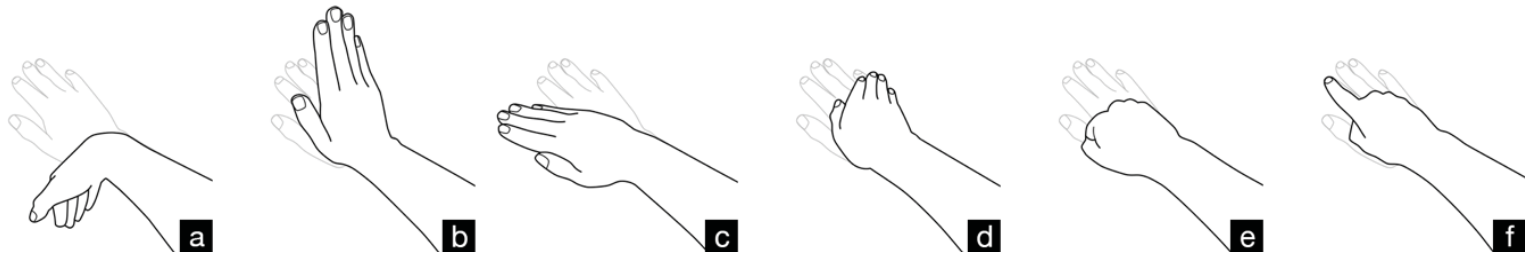

Fig. 4. Illustration of the gestures used in the study. a) wrist flexion, b) wrist extension, c) radial deviation, d) ulnar deviation, e) squeeze fingers, f) lift index finger.

Participants were standing during the study and wore the sleeve on their dominant forearm. The participants were instructed to hold their hand over a table in front of them, with their elbow bent at 90 degrees. While this may introduce some constant muscle tension, the effects of this would be removed by the normalization step in the EMG reading process. Participants advanced through the study with a wireless keyboard and mouse. The laptop running the stimulation hardware was kept out of reach, preventing any common ground concerns.

On-screen instructions guided the participants through the study. Initially, they were asked to manually perform the gestures, during which EMG data was gathered. Upon completing the manual performance and data collection part of the study, the participants moved on to the pose-replay portion of the study. In a randomized order, the EMS stimulation pattern for each gesture was computed from the EMG data. Once the stimulation pattern was calculated, it was displayed to the participants in an on-screen GUI. The participants were then free to explore the amplitude, frequency and pulse width of the stimulation using the keyboard. Initial frequency $(55 \mathrm{~Hz})$ and pulse width $(200 \mu \mathrm{s})$ values were configured. The use of the keyboard allowed fine-grained control of each parameter and provided a quick method to disable the stimulation (by pressing the spacebar). The participants were free to spend as long as they wished exploring the parameters. During exploration of the stimulation pattern, the participants' wrist, back of hand, and fingers were tracked with an OptiTrack motion tracking camera setup (8 Prime13 cameras, 240fps). Data was captured at the end of every complete line of reading (EMG), and after every 5th step of stimulation amplitude increase (EMS). This resulted in 18 frames of data per pose during the reading phase, and 24 frames per pose (on average) during the writing phase. The captured data was automatically inspected after each participant completed the study. The motion tracking data was used to calculate orientation and position changes of the back of the hand and the individual fingers during poses (manual and stimulated).

The target gestures were grouped into pairs based on shared muscle compartments: wrist-up and wristdown, hand-left and hand-right, squeeze-fingers and index-point. For example, the extensor carpi ulnaris (on the top of the forearm), among other muscles, contracts to extend the wrist. In this case, it is an agonist muscle. The antagonist muscles, such as the flexor carpi ulnaris (on the bottom of the forearm), relax to enable wrist extension. When flexing the wrist, however, the role of these muscles is reversed. When holding a pose, co-activation of both agonist and antagonist muscles can occur [4,24], producing discernible signals for our EMG devices. Therefore, we pair gestures in our analysis to explore the correct identification of active muscle compartments.

\subsection{Results}

The participants performed 54 gestures in total. Wrist-down and Hand-right were the most accurately reproduced poses, with $89 \%$ accuracy. This means that, at some time during exploration of the EMS pattern, the back of hand and/or fingers were stimulated to move in the target direction. For both of these poses, the 
error case moved in the direction of the target's opposing movement, Wrist-up and Hand-left, respectively. Index-Point was the next most accurately reproduced, with $67 \%$ accuracy. The index-point errors all resulted in a Wrist-Up pose. Hand-left was the least accurate pose, with only $11 \%$ accuracy. The majority of the HandLeft stimulation patterns resulted in a Wrist-Down pose. Squeeze-Fingers was the second least accurate, with $33 \%$ accuracy.

On average, $28(52 \%)$ of the gestures were replayed correctly. $8(15 \%)$ of the EMS gestures moved in the direction of the target's pair (i.e. favoring the antagonist muscles). For example, four instances of the wrist-up gesture moved in the hand-down direction. 15 (28\%) of the gestures moved in a wrong axis, and 3 (6\%) instances resulted in no movement at all.

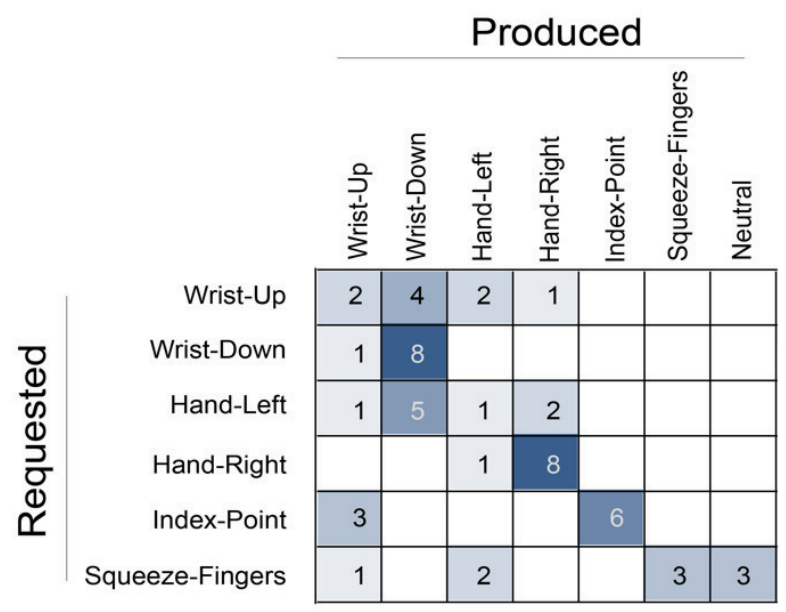

Fig. 5. Confusion matrix of study results. In total, $52 \%$ of gestures were replayed correctly, with Wrist-Down and HandRight both achieving $89 \%$ replay accuracy.

\section{DISCUSSION}

EMS in HCI is currently restricted by two challenges. First, it is low resolution, supporting only coarse movements across a limited range of gestures and preventing opportunities for complex interaction techniques. Second, it requires expert calibration to determine correct electrode positioning and signal parameters. High density electrode arrays can both increase stimulation resolution, better supporting finer movements, and improve comfort and reduce fatigue. But they are complex to calibrate and are yet to be adopted in HCI. We explore EMG as a method of auto-calibrating EMS arrays. We are the first to explore auto-calibration of complex stimulation patterns in EMS arrays. Through this exploration, we seek to make high resolution EMS available to the HCI community, without the need for complex and time-consuming calibration procedures.

We achieve $52 \%$ replay accuracy. Across the three most accurate poses, we achieve $82 \%$ replay accuracy. The most accurate poses (Hand-Right and Wrist-Down) are replayed with $89 \%$ accuracy. In both of these instances, the error case moved in the direction of the pose's antagonist muscle. Holding a pose can lead to agonist-antagonist co-activation [4,24] and therefore create EMG signals across the two muscle compartments. We successfully identified these muscle compartments in $67 \%$ of gestures. These results demonstrate the potential of EMG to auto-calibrate EMS arrays and motivate future work to explore calibration of increasingly complex gestures.

Our current calibration procedure takes approximately 1 minute per pose (45 seconds to gather EMG data and 15 seconds for amplitude exploration.) When successful, this represents a practical improvement over the 
2-5 minutes reported in Affordance++. Furthermore, the relative success of our 'index-point' gesture (Fig. 5), the most complex pose in our study in terms of muscle recruitment ${ }^{5}$, demonstrates that calibration of advanced poses can be achieved with the EMG-calibration technique with no increase in calibration time. Although this decrease in calibration time may seem incremental at this stage, this improvement quickly scales as further gestures are added to an interaction technique.

\subsection{Increasing the Accuracy of Auto-Calibration}

Our study results demonstrate the potential of auto-calibrating EMS arrays with EMG. We now discuss ways in which these results can be improved further still.

In $67 \%$ of gestures, our EMG-EMS mapping correctly identified the muscle compartments involved in performing the gesture. When holding a pose, co-activation can cause EMG potentials across the two muscle compartments. However, where agonist muscle potentials increase over time, antagonist muscles do not [30]. Better modelling of agonist-antagonist behaviors, such as monitoring changes in certain electrodes over time, could improve the overall accuracy of the proposed calibration technique.

The least successful gesture was Hand-Left, with only $11 \%$ accuracy, or one correct replay. A principle muscle in the production of this gesture, also called a radial deviation, is the flexor carpi radialis. This muscle runs up the center underside of the forearm, roughly in-line with typical positioning of the zip (see Fig. 1b). For this reason, the electrodes proximal to the zip would have measured comparatively high EMG potentials, due to skin surface capacitance, while being physically positioned over neighboring muscles. Any stimulation applied through those electrodes would have stimulated neighboring muscles, in this case those involved in our Wrist-down gesture. This can be seen as 55\% of the Hand-left gestures resulted in a Wrist-down motion. It is important, therefore, to consider areas of the skin that are not covered by electrodes, such as underneath fabric fasteners. The effect of these areas on calibration accuracy is two-fold. Firstly, as those muscles are not covered by an electrode, they cannot be directly targeted with stimulation. Secondly, without electrodes directly over the muscles to record high potentials, the neighboring electrodes may appear comparatively high, resulting in their selection for stimulation. This will likely lead to inaccurate stimulation results.

In our study, participants increased one channel of amplitude to explore the generated pattern. This channel automatically maintained a calculated ratio to the amplitude of the second channel (based on differences in measured EMG potentials). This may have had a negative impact on our results. Anecdotally, after participating in the study, one participant re-visited the stimulation patterns with individuallycontrollable channel amplitudes. This participant was able to correctly perform, or improve, the accuracy of certain stimulation patterns with this technique. This suggests the need for a further exploration of the automatically calculated amplitude ratios.

The participants also did not vary the pulse widths and frequencies per channel, but instead used the predefined values $(55 \mathrm{~Hz}$ and $200 \mu \mathrm{s})$. As varying parameters can target different muscle depths, this can result in increased accuracies for given stimulation patterns. Although automatically determining stimulation frequencies from the EMG data remains an exciting prospect, this would require a significantly higher sampling rate than we currently achieve.

As electrodes spanned the entirety of the forearm, many of the generated stimulation patterns utilized electrodes closer to the wrist than previously explored. One reason for this may be the reading direction of the sleeve - from the top of the sleeve to the bottom of the sleeve. During sub-maximal contraction, EMG amplitudes increase over time [30]. This favors the electrodes towards the wrist, increasing their likelihood of selection for stimulation. It may be possible to reduce the impact of this by randomizing the order in which electrodes are read across the sleeve. Another approach may be to take sparsely-sampled repeat readings, from which to normalize the EMG amplitudes across the sleeve.

\footnotetext{
${ }_{5}^{5}$ The 'index point' gestures involves folding three fingers and extending a fourth. This is achieved by isolating the individual finger 'slips' of the underlying shared muscle groups. See [35] for an anatomical overview.
}

Proceedings of the ACM on Interactive, Mobile, Wearable and Ubiquitous Technologies, Vol. 1, No. 3, Article 68. Publication date: September 2017 
The accuracy of the calibration process results from the accuracy of the EMG data. In our prototype, there is a distance of approximately $1.5 \mathrm{~m}$ between the EMG source and the signal amplification circuitry. By moving the amplifiers closer to the source of the signal, perhaps embedding them into the sleeve itself in our case, we may improve our EMG data resolution. Similarly, De Marchis et al., in their work on 1:1 electrode calibration, use $5 \mathrm{kHz}$ EMG and a 16-bit analog to digital converter (ADC). We do not achieve that data rate, nor that resolution of data conversion, from our Arduino Mega. Improving these factors would increase our EMG resolution and enable higher calibration accuracy.

Although our calibration time represents an improvement over existing techniques, 1 minute-per-pose is not the lowest time bound for this technique. The EMG devices used in our study perform raw signal processing and windowing on-board, and, due to the spike in the signal when switching relays, result in the $220 \mathrm{~ms}$ read time per electrode pair. By moving raw signal processing to software (as in e.g. [25]), we can reduce our electrode pair read time from $220 \mathrm{~ms}$ to $\sim 50 \mathrm{~ms}$ without loss of data resolution. This brings our perpose calibration time closer to 30 seconds with two off-the-shelf devices (15 seconds of data capture, 15 seconds of amplitude exploration). With more devices, this can be reduced up to the point where muscles can be read and electrode patterns calculated in interactive time.

When successful, our calibration time represents an improvement over existing manual techniques. With the proposed speed up changes, above, our calibration time can equal or improve upon that of UnlimitedHand [32]. When unsuccessful, however, there are few options to then successfully calibrate the sleeve. Given the noise in EMG sensing (such as the previously discussed sensing resolution), the user could re-run the EMG calibration process, however there is no guarantee of success here. Otherwise, our multi-electrode stimulation style requires many $\left(\sim 10^{18}\right)$ steps for manual calibration. Anecdotally, after the study, some participants were given a basic GUI for selecting electrodes and controlling stimulation parameters, in order to further explore the capabilities of the sleeve. We expected the participants to consider their underlying musculature and make informed electrode selections, but we saw no evidence of this. Instead, participants would make seemingly random selections to 'see what happened'. Even with the underlying anatomical knowledge, the authors were no more successful in manually calibrating the participants, in part due to the underlying anatomical differences, and in part due to the lack of haptic and motor feedback.

We read between each electrode and its six neighboring electrodes. This is in order to improve reading accuracy along muscles that do not tend directly down the arm (pennate muscles, for example). This adds a complexity to our mapping procedure, as at any given electrode location, we do not know which electrode pair to favor. While we assume that electrodes directly down the muscle body will provide the highest readings, this is a potential source for noise and error in our modeling. Given the fact that we cannot determine the muscle structure under our electrodes, we believe it would be beneficial to move from a bipolar EMG configuration to a monopolar configuration (gathering EMG data from individual electrodes). This has recently been shown to offer increased accuracy over a bipolar approach when processing data from the $5^{\text {th }}$ principle component [31].

As with the calibration discussed in Affordance++, the approach presented in this paper is a per-pose technique. The technique presented by Tamaki et al. in PossessedHand is not a per-pose approach, rather is an all-possible-movements approach [33]. From their calibration data, Tamaki et al. could calculate the stimulation required to achieve any desired pose (within their possible joint-selection space). This is evidently advantageous, as not all desired gestures need to be designed 'ahead of time,' and yet no underlying muscle knowledge is required. As previously discussed, Tamaki et al.'s random walk technique cannot practically scale to electrode arrays. By increasing the number of EMG devices used and therefore approaching real-time EMG tracking, the EMG-calibration technique presented here could be moved from a per-pose to an allpossible-movements approach. For example, the user could put on the sleeve, and wiggle their fingers and roll their wrist in a specified pattern, while EMG data is collected, in order to arrive at a complex calibration mapping. Stimulation amplitudes would still require exploration; however, this could then be explored as an iterative loop between EMS and EMG in real time, with EMG readings being taken between EMS stimulation pulses.

Proceedings of the ACM on Interactive, Mobile, Wearable and Ubiquitous Technologies, Vol. 1, No. 3, Article 68. Publication date: September 2017. 


\section{CONCLUSION}

Current EMS in HCI is limited by both calibration complexity and low resolution. As we seek to address the resolution by using increasing numbers of electrodes, the complexity of calibration scales exponentially. The medical literature has shown that EMS arrays can offer increased stimulation resolution over traditional dual electrode approaches, but a calibration technique capable of maximizing their potential is yet to be provided. We explore EMG as a method to spatially calibrate these arrays, selecting the complex arrangement of correct electrodes to achieve desired movements. We detail a study in which 9 participants performed 6 gestures while EMG data was gathered, and were then stimulated to re-perform these gestures. We report $81.7 \%$ accuracy across our three most accurate gestures. On average, we achieve 52\% EMS-gesture accuracy and 15\% antagonist stimulation, resulting in movement in the opposite direction. In combination, this shows $67 \%$ correct muscle group identification. Our study incorporated all of the gestures frequently seen across the HCI EMS literature without (a) the need for any reconfiguration or (b) expertise for calibration. This demonstrates the potential of this technique.

Our spatial calibration procedure requires $\sim 1$ minute per pose, representing an improvement over techniques discussed in the literature (e.g., [18]). Furthermore, the time requirement per pose does not increase with pose complexity. This time-per-pose does not represent the lower temporal bound of this technique, however, and with further hardware improvements, this technique can approach real-time. Additionally, with improved circuitry and better agonist-antagonist modelling, further accuracy can also be attained. Our work lays a promising foundation upon which further research can build to address the spatial calibration problem, facilitating higher resolution stimulation and opportunities for out-of-the-lab EMS exploration. Through this exploration, we may come to use EMS in increasingly ubiquitous and pervasive settings.

\section{REFERENCES}

[1] Christoph Amma, Thomas Krings, Jonas Böer, and Tanja Schultz. 2015. Advancing Muscle-Computer Interfaces with High-Density Electromyography. [In Proceedings of the 33rd Annual ACM Conference on Human Factors in Computing Systems (CHI '15), 929-938]. https://doi.org/10.1145/2702123.2702501

[2] Christopher Assad, Michael Wolf, Theodoros Theodoridis, Kyrre Glette, and Adrian Stoica. 2013. BioSleeve: A Natural EMG-based Interface for HRI. [In Proceedings of the 8th ACM/IEEE International Conference on Human-robot Interaction (HRI '13), 69-70]. Retrieved September 18, 2016 from http://dl.acm.org/citation.cfm?id=2447556.2447571

[3] Chad E. Bouton, Ammar Shaikhouni, Nicholas V. Annetta, Marcia A. Bockbrader, David A. Friedenberg, Dylan M. Nielson, Gaurav Sharma, Per B. Sederberg, Bradley C. Glenn, W. Jerry Mysiw, Austin G. Morgan, Milind Deogaonkar, and Ali R. Rezai. 2016. Restoring cortical control of functional movement in a human with quadriplegia. Nature 533, 7602: 247-250]. https://doi.org/10.1038/nature17435

[4] Shyang Chang, Ming-Chun Hsyu, Hsiu-Yao Cheng, Sheng-Hwu Hsieh, and Chen-Chiang Lin. 2008. Synergic Co-activation in Forearm Pronation. Annals of Biomedical Engineering 36, 12: 2002-2018]. https://doi.org/10.1007/s10439-008-9569-x

[5] Cristiano De Marchis, Thiago Santos Monteiro, Cristina Simon-Martinez, Silvia Conforto, and Alireza Gharabaghi. 2016. Multicontact functional electrical stimulation for hand opening: electrophysiologically driven identification of the optimal stimulation site. Fournal of NeuroEngineering and Rehabilitation 13]. https://doi.org/10.1186/s12984-016-0129-6

[6] Tim Duente, Max Pfeiffer, and Michael Rohs. 2016. On-skin Technologies for Muscle Sensing and Actuation. [In Proceedings of the 2016 ACM International foint Conference on Pervasive and Ubiquitous Computing: Adjunct (UbiComp '16), 933-936]. https://doi.org/10.1145/2968219.2979136

[7] C Frigo, M Ferrarin, W Frasson, E Pavan, and R Thorsen. 2000. EMG signals detection and processing for on-line control of functional electrical stimulation. Fournal of Electromyography and Kinesiology 10, 5: 351-360]. https://doi.org/10.1016/S10506411(00)00026-2

[8] Marco Gazzoni, Nicolò Celadon, Davide Mastrapasqua, Marco Paleari, Valentina Margaria, and Paolo Ariano. 2014. Quantifying Forearm Muscle Activity during Wrist and Finger Movements by Means of Multi-Channel Electromyography. PLOS ONE 9, 10: e109943]. https://doi.org/10.1371/journal.pone.0109943

[9] Manami Katoh, Narihiro Nishimura, Maki Yokoyama, Taku Hachisu, Michi Sato, Shogo Fukushima, and Hiroyuki Kajimoto. 2013. Optimal Selection of Electrodes for Muscle Electrical Stimulation Using Twitching Motion Measurement. [In Proceedings of the 4th Augmented Human International Conference (AH '13), 237-238]. https://doi.org/10.1145/2459236.2459279

[10] Oliver Beren Kaul, Max Pfeiffer, and Michael Rohs. 2016. Follow the Force: Steering the Index Finger Towards Targets Using EMS. [In Proceedings of the 2016 CHI Conference Extended Abstracts on Human Factors in Computing Systems (CHI EA '16), 2526-2532]. https://doi.org/10.1145/2851581.2892352 
68:16 J. Knibbe et al.

[11] T. Keller, M. Lawrence, A. Kuhn, and M. Morari. 2006. New Multi-Channel Transcutaneous Electrical Stimulation Technology for Rehabilitation. [In 28th Annual International Conference of the IEEE Engineering in Medicine and Biology Society, 2006. EMBS '06, 194-197]. https://doi.org/10.1109/IEMBS.2006.259399

[12] Thierry Keller and Andreas Kuhn. 2008. Electrodes for transcutaneous (surface) electrical stimulation. fournal of Automatic Control 18, 2: 35-45].

[13] Andreas Kuhn, Thierry Keller, Silvestro Micera, and Manfred Morari. 2009. Array electrode design for transcutaneous electrical stimulation: a simulation study. Medical Engineering \& Physics 31, 8: 945-951]. https://doi.org/10.1016/j.medengphy.2009.05.006

[14] Pedro Lopes and Patrick Baudisch. 2013. Muscle-propelled Force Feedback: Bringing Force Feedback to Mobile Devices. [In Proceedings of the SIGCHI Conference on Human Factors in Computing Systems (CHI '13), 2577-2580]. https://doi.org/10.1145/2470654.2481355

[15] Pedro Lopes, Lars Butzmann, and Patrick Baudisch. 2013. Muscle-propelled Force Feedback: Bringing Force Feedback to Mobile Devices Using Electrical Stimulation. [In Proceedings of the 4th Augmented Human International Conference (AH '13), 231-232]. https://doi.org/10.1145/2459236.2459276

[16] Pedro Lopes, Alexandra Ion, and Patrick Baudisch. 2015. Impacto: Simulating Physical Impact by Combining Tactile Stimulation with Electrical Muscle Stimulation. [In Proceedings of the 28th Annual ACM Symposium on User Interface Software \& Technology (UIST '15), 11-19]. https://doi.org/10.1145/2807442.2807443

[17] Pedro Lopes, Alexandra Ion, Willi Mueller, Daniel Hoffmann, Patrik Jonell, and Patrick Baudisch. 2015. Proprioceptive Interaction. [In Proceedings of the 33rd Annual ACM Conference on Human Factors in Computing Systems (CHI '15), 939-948]. https://doi.org/10.1145/2702123.2702461

[18] Pedro Lopes, Patrik Jonell, and Patrick Baudisch. 2015. Affordance++: Allowing Objects to Communicate Dynamic Use. [In Proceedings of the 33rd Annual ACM Conference on Human Factors in Computing Systems (CHI '15), 2515-2524]. https://doi.org/10.1145/2702123.2702128

[19] Pedro Lopes, Doăa Yüksel, François Guimbretière, and Patrick Baudisch. 2016. Muscle-plotter: An Interactive System Based on Electrical Muscle Stimulation That Produces Spatial Output. [In Proceedings of the 29th Annual Symposium on User Interface Software and Technology (UIST '16), 207-217]. https://doi.org/10.1145/2984511.2984530

[20] Nebojša M. Malešević, Lana Z. Popović Maneski, Vojin Ilić, Nikola Jorgovanović, Goran Bijelić, Thierry Keller, and Dejan B. Popović. 2012. A multi-pad electrode based functional electrical stimulation system for restoration of grasp. Journal of NeuroEngineering and Rehabilitation 9: 66]. https://doi.org/10.1186/1743-0003-9-66

[21] Nebojsa Malesevic, Lana Popovic, Goran Bijelic, and Goran Kvascev. 2010. Muscle twitch responses for shaping the multi-pad electrode for functional electrical stimulation. Journal of Automatic Control 20: 53-58].

[22] S. Mathur, J. J. Eng, and D. L. MacIntyre. 2005. Reliability of surface EMG during sustained contractions of the quadriceps. fournal of Electromyography and Kinesiology 15, 1: 102-110]. https://doi.org/10.1016/j.jelekin.2004.06.003

[23] Jess McIntosh, Charlie McNeill, Mike Fraser, Frederic Kerber, Markus Löchtefeld, and Antonio Krüger. 2016. EMPress: Practical Hand Gesture Classification with Wrist-Mounted EMG and Pressure Sensing. [In Proceedings of the 2016 CHI Conference on Human Factors in Computing Systems (CHI '16), 2332-2342]. https://doi.org/10.1145/2858036.2858093

[24] Jeremy Mogk and Peter Keir. 2003. The effects of posture on forearm muscle loading during gripping. Ergonomics 46, 9: 956-975]. https://doi.org/10.1080/0014013031000107595

[25] Jun Nishida and Kenji Suzuki. 2016. bioSync: Synchronous Kinesthetic Experience Among People. [In Proceedings of the 2016 CHI Conference Extended Abstracts on Human Factors in Computing Systems (CHI EA '16), 3742-3745]. https://doi.org/10.1145/2851581.2890244

[26] Max Pfeiffer, Tim Dünte, Stefan Schneegass, Florian Alt, and Michael Rohs. 2015. Cruise Control for Pedestrians: Controlling Walking Direction Using Electrical Muscle Stimulation. [In Proceedings of the 33rd Annual ACM Conference on Human Factors in Computing Systems (CHI '15), 2505-2514]. https://doi.org/10.1145/2702123.2702190

[27] Dejan B. Popović and Mirjana B. Popović. 2011. Advances in the use of electrical stimulation for the recovery of motor function. Progress in Brain Research 194: 215-225]. https://doi.org/10.1016/B978-0-444-53815-4.00005-4

[28] Ana Popović-Bijelić, Goran Bijelić, Nikola Jorgovanović, Dubravka Bojanić, Mirjana B. Popović, and Dejan B. Popović. 2005. MultiField Surface Electrode for Selective Electrical Stimulation. Artificial Organs 29, 6: 448-452]. https://doi.org/10.1111/j.15251594.2005.29075.x

[29] Raafat Shalaby, Thomas Schauer, Wolfgang Liedecke, and Jörg Raisch. 2011. Amplifier design for EMG recording from stimulation electrodes during functional electrical stimulation leg cycling ergometry. Biomedizinische Technik. Biomedical Engineering 56, 1: 2333]. https://doi.org/10.1515/BMT.2010.055

[30] Didier Staudenmann, Jaap H. van Dieën, Dick F. Stegeman, and Roger M. Enoka. 2014. Increase in heterogeneity of biceps brachii activation during isometric submaximal fatiguing contractions: a multichannel surface EMG study. Fournal of Neurophysiology 111, 5: 984-990]. https://doi.org/10.1152/jn.00354.2013

[31] Didier Staudenmann, Dick F. Stegeman, and Jaap H. van Dieën. 2013. Redundancy or heterogeneity in the electric activity of the biceps brachii muscle? Added value of PCA-processed multi-channel EMG muscle activation estimates in a parallel-fibered muscle. Journal of Electromyography and Kinesiology: Official Journal of the International Society of Electrophysiological Kinesiology 23, 4: 892-898]. https://doi.org/10.1016/j.jelekin.2013.03.004

[32] Emi Tamaki, Terence Chan, and Ken Iwasaki. 2016. UnlimitedHand: Input and Output Hand Gestures with Less Calibration Time. [In Proceedings of the 29th Annual Symposium on User Interface Software and Technology (UIST '16 Adjunct), 163-165]. https://doi.org/10.1145/2984751.2985743

Proceedings of the ACM on Interactive, Mobile, Wearable and Ubiquitous Technologies, Vol. 1, No. 3, Article 68. Publication date: September 2017. 
[33] Emi Tamaki, Takashi Miyaki, and Jun Rekimoto. 2011. PossessedHand: Techniques for Controlling Human Hands Using Electrical Muscles Stimuli. [In Proceedings of the SIGCHI Conference on Human Factors in Computing Systems (CHI '11), 543-552]. https://doi.org/10.1145/1978942.1979018

[34] M. T. Wolf, C. Assad, A. Stoica, Kisung You, H. Jethani, M. T. Vernacchia, J. Fromm, and Y. Iwashita. 2013. Decoding static and dynamic arm and hand gestures from the JPL BioSleeve. [In 2013 IEEE Aerospace Conference, 1-9]. https://doi.org/10.1109/AERO.2013.6497171

[35] 1. The Flexors And Extensors Of The Fingers. Retrieved May 2, 2017 from http://chestofbooks.com/health/anatomy/Human-BodyConstruction/1-The-Flexors-And-Extensors-Of-The-Fingers.html

Received February 2017; revised April 2017; accepted June 2017

Proceedings of the ACM on Interactive, Mobile, Wearable and Ubiquitous Technologies, Vol. 1, No. 3, Article 68. Publication date: September 2017 